\title{
Empatía y cuidado profesional: esbozos de una relación problemática
}

\author{
The role of empathy in professional care
}

Patricia Olivares ${ }^{1}$

\begin{abstract}
Resumen
Introducción: tres paradigmas actuales acerca del cuidado profesional en medicina sitúan a la empatía como la actitud básica necesaria para lograr un cuidar de otros que respete la dignidad de las personas atendidas en los servicios de salud. Sin embargo, los conceptos de empatía difieren en estos paradigmas, algo que parece ser reflejo de la confusión general que existe respecto a esta noción. Por otro lado, hay quienes critican el que se pretenda reducir a ella la actitud fundamental del cuidar de otros. Además de esto, existen formas de comprender la medicina que, eventualmente. Métodos: a partir de estos tres paradigmas de cuidado, se profundiza en las teorías acerca de la empatía más importantes de la actualidad. El método que se utiliza es de análisis filosófico de los datos provenientes de las formas de comprender el cuidado profesional. Resultados: existen formas muy distintas de comprender la noción de empatía no solo desde paradigmas que gravitan en torno al cuidado profesional, sino también dentro de la filosofía. Las comprensiones de la noción son incluso disímiles, lo que hace imposible avanzar en la enseñanza y en el diálogo con respecto a la posición de la empatía en el cuidado. Se propone la noción fenomenológica de empatía que la sitúa en la esfera de las facultades espirituales del sujeto. Conclusiones: la empatía es un elemento relevante del cuidado profesional, pero parece no poder situarse en el centro del mismo como actitud básica. Es una herramienta útil que podría ser enseñada y evaluada en la educación médica, siempre y cuando se trabaje con una noción unívoca de ella.
\end{abstract}

Palabras clave: cuidado; empatía; cariño; cuidado centrado en el paciente; cuidado integral del paciente

\begin{abstract}
Introduction: Abstract Introduction: Three current paradigms of professional health services situate empathy as the fundamental attitude necessary to respects the dignity of those serve. However, current concepts of empathy differ widely in these paradigms, something that seems to reflect the general confusion regarding this notion. On the other hand, there are those who criticize the attempt to reduce to it to the fundamental attitude of caring for others. Methods: From these three paradigms of healthcare, the most important current theories of empathy will be treated. The method employs a philosophical analysis of the data coming from the ways of understanding professional care. Results: there are very different ways of understanding the notion of empathy not only in healthcare, 9 but in philosophy as well. The understanding of the notion varies greatly, making it impossible to reach consensus in teaching and dialogue with respect to the role of empathy in health care. The proposal is to use a phenomenological approach to empathy which places it in the sphere of the subject's spiritual faculties. Discussion: empathy is a relevant element of professional healthcare, but not central as a fundamental attitude. It is a useful tool that could be taught and evaluated in medical education, as long as it is applied with a common understanding.
\end{abstract}

Keywords: care; empathy; caring; patient centered care; whole person care.

Fecha de envío: 5 de noviembre de 2019 - Fecha de aceptación: 19 de junio de 2020

\section{Introducción}

En el ámbito de la atención de la salud, la empatía es central en los tres paradigmas más importantes en la discusión actual acerca del cuidado: Cuidado centrado en el paciente/cliente
(Patient/Client Centered Care), la ética del cuidado y el cuidado integral de la persona (Whole Person Care). Así, por ejemplo, Carl Rogers, que fue quien acuñó el término cuidado centrado en el cliente (Client Centered Care), se refiere a la empatía en estos términos:

(1) Centro de Bioética, Pontificia Universidad Católica de Chile.

Autor de correspondencia:pcolivar@uc.cl 
"Sentir el mundo privado del cliente como si fuera el suyo propio, pero sin perder nunca la cualidad de "como si", es empatía, y esto parece esencial para la terapia. Sentir la ira, el miedo o la confusión del cliente como si fuera el suyo, pero sin que su propia ira, miedo o confusión se vea envuelta en ella, es la condición que nos esforzamos por describir" (Rogers, 1957)

A pesar del uso de la palabra "sentir", en su obra más conocida, Psicoterapia centrada en el cliente, Rogers describe la actitud empática en términos de "percepción"y contrapone la identificación emocional del consejero con la identificación empática:

“(...) la experiencia con el cliente, la vivencia de sus actitudes, no se da en términos de identificación emocional por parte del consejero, sino más bien como una identificación empática, por la que el consejero percibe los odios, las esperanzas y temores del cliente a través de la inmersión en un proceso empático, pero sin que él mismo experimente esos odios, esperanzas y temores." (Rogers, 1981)

La identificación empática en Rogers se da cuando se abandona la tendencia a hacer evaluaciones y diagnósticos y el terapeuta se concentra en proporcionar al cliente una profunda comprensión y aceptación de las actitudes manifestadas por él en el momento de la entrevista (Rogers, 1981).

Por su parte, Nel Noddings, una de las primeras teóricas de la ética del cuidado identifica la relación de cuidado con la empatía. En sus palabras:

"Cuidar implica, para el cuidador, un "sentir con" el otro. Llamaremos a esta relación: "empatía", pero reflexionaremos acerca de lo que significa este término [...] la noción de "sentir con" que estoy delineando no implica proyección, sino recepción. Lo he llamado "estar absorto". No"me pongo en los zapatos de otro", por así decir, para analizar su realidad como dato objetivo y para preguntarme: ¿cómo me sentiría en esta situación? Por el contrario, resisto la tentación de analizar y planear. No me proyecto, recibo al otro en mí mismo y veo y siento con el otro" (Noddings, 2013)

Para Noddings la empatía es claramente la actitud interna o la disposición interior que hace posible el cuidar de otros. Además, incluye una sintonía emocional en la que no solo se llega a tener noticia del sentimiento del otro, sino además"siento con el otro lo que él siente". Este sentimiento no es producto de una proyección de la propia vida emocional en la situación del otro, sino de un "recibir" sus sentimientos en mí. Esta recepción se produce por un proceso de captación no deductivo.
Asimismo, la empatía es una actitud fundamental del cuidado integral de la persona. Así, para Hutchinson, para quien la sanación es un viaje del paciente en el cual el terapeuta es un acompañante, el "ponerse en los zapatos del otro" es la forma en que se concreta esa compañía (Hutchinson, 2017). Para Fricchione, "la empatía unida a la confianza son las claves para mantener el alma de la medicina" (Fricchione, 2017). En el consenso nacional e internacional para desarrollar el ámbito de la espiritualidad en el enfoque del cuidado integral de la persona, se menciona la necesidad de

“Comprender y empatizar con las diversas, pero a menudo sutiles costumbres culturales que influyen en las creencias y prácticas espirituales en todo el mundo"(Puchalski et al., 2014).

"Sentir el mundo privado del otro"," ponerse en los zapatos del otro", "sentir con el otro", ¿son términos equivalentes? ¿Se están refiriendo estos autores a la misma noción? ¿Qué se está queriendo decir al afirmar que la empatía es central en la relación terapéutica? Estas son las preguntas que se abordarán en lo que sigue.

\section{Materiales y Método}

Partiendo de la idea de la empatía como un aspecto central del cuidado profesional, tal como lo afirman los paradigmas de cuidado que se utilizan actualmente en la práctica clínica, se realizó un análisis filosófico de las teorías más relevantes acerca de la empatía. La reflexión se acotó por medio del atenerse a los paradigmas de empatía en medicina clínica, excluyendo aquellos, que, aunque relevantes para la fenomenología o el estudio de las emociones, no parecen tener influencia directa en este ámbito. Se excluyen también los aportes de pensadores relevantes en el ámbito de la siquiatría, debido a que no han permeado en la práctica clínica del resto de las especialidades más, por decir así, "biomédicas". En los resultados, se desarrolla, con mayor detalle, la teoría que parece proveer el fundamento más adecuado a las intuiciones que provienen de los paradigmas de cuidado.

\section{Antecedentes de la noción de empatía}

La noción moderna de empatía tiene una primera vertiente en la idea de "simpatía" de David Hume y Adam Smith. Hume escribe usando la palabra sympathy, pero dado su contenido, autores como Jesse Prinz han identificado sympathy con la palabra empatía. Para este filósofo, en interpretación de Prinz, sympathy, es "una inferencia asociativa a partir de expresiones de emoción observadas o imaginadas que son conocidas por la experiencia y producen emociones" (Prinz, 2011). Es decir, cuando alguien ve el gesto dolor de otra persona la mente formaría la idea del dolor y eso produciría la misma emoción en el observador. Por su parte, 
Adam Smith parece haber sido un poco más ambiguo en su noción de empatía. Amy Coplan, comentando a Smith refiere que, en ocasiones, este ve la empatía como un proceso imaginativo en el cual el observador se supone a sí mismo en la situación de otro, ("¿cómo sería ser él?"), mientras que, en otras ocasiones, afirma que se trata de imaginar los propios sentimientos en el caso de estar en el lugar del otro ("¿cómo me sentiría yo si me pasara lo mismo que a él?) (Coplan \& Goldie, 2014).

Por otro lado, como palabra, empatía proviene de la palabra alemana Einfühlung, que fue utilizada por primera vez, en el ámbito de la estética, por Robert Visher y asumida en 1873 por Theodore Lipps (Zahavi, 2014a). Este último conceptualiza la empatía en el marco de aquello que permite el conocimiento de otros como una proyección de la propia vida psíquica en los otros sujetos, es decir, una suerte de penetrar y cubrir la vida psíquica ajena con la propia psiquis (Zahavi, 2014a). Es decir, sentir empatía sería experimentar una parte de la propia vida psíquica como perteneciente a otro. Por eso le llama "conocimiento sui generis". Es de notar que, sin embargo, según Lipps, solo conocemos nuestros propios estados mentales, jamás los de los demás, los cuales solo podemos simular. (Zahavi, 2014b).

Por otro lado, un hecho relevante para este tema fue el descubrimiento de las "neuronas espejo". En la década de los 80, Rizzolatti y di Pellegrino describieron un grupo de neuronas en el cerebro del macaco que descargaban tanto cuando el mono realizaba una acción dirigida a una meta con las manos (agarrar, sostener, manipular), como cuando observaba que otro mono, o el investigador, realizaban la misma acción (di Pellegrino et al., 1992). Dada la localización de estas neuronas en una zona del cerebro del macaco asimilable con el área de Brocca, estos autores elaboraron la hipótesis de que este hecho desempeña un papel en la interpretación de los eventos motores. Los subsecuentes descubrimientos de neuronas espejo en otras áreas del cerebro de los macacos y de un sistema similar en el cerebro humano dieron pie a que se las identificara como la base estructural de la empatía y de la comunicación interpersonal. En este punto el optimismo con respecto a la empatía no conoció ningún límite, se comparó descubrimiento de las neuronas espejo con el de la doble hebra helicoidal de ADN. Y, sin embargo, tal como mostraré a continuación, existe una gran confusión con respecto a la noción de empatía. Sin una debida clarificación, estos hallazgos y su aplicabilidad a la realidad se reducen enormemente.

Las corrientes de pensamiento descritas se podrían ordenar en base a dos coordenadas. Una primera coordenada sería asumir que la conciencia es accesible por medio de instrumentos técnicos como podrían ser las neuroimágenes funcionales. La segunda gira en torno a la posibilidad de acceder a la conciencia ajena de modo directo o indirecto. La teoría de las neuronas espejo sería un ejemplo de la primera coordenada, mientras que las ideas de Smith, Hume y Lipps son ejemplos de una postura que considera que el acceso a la conciencia ajena se realiza de modo indirecto, por simulación, pero en definitiva, sin acceso real a la conciencia de otros. La tercera alternativa dentro de la segunda coordenada, es decir, el acceso directo a la conciencia ajena es la que postularon en su tiempo, Husserl, Stein y Scheler entre otros. Sin negar el valor de los hallazgos que se puedan realizar con neuroimágenes, ni el que la comunicación interpersonal tenga elementos de simulación, afirmo que en el encuentro real entre terapeuta y paciente puede producirse un acceso directo a la conciencia ajena y, aún más, que es gracias a ese acceso directo que el terapeuta puede comprender y acoger al paciente. Dicho de otro modo, toda vez que en la relación terapéutica logramos comprender al paciente y él se siente comprendido por nosotros, lo que algunos Ilaman coloquialmente "conectar" con el paciente, se debe al acceso directo a la conciencia del paciente y viceversa. Como terapeuta, no solo entiendo racionalmente que el otro sufre, sino que conecto con ese sufrimiento. A su vez, el paciente se siente acogido cuando conecta con una respuesta adecuada a su sufrimiento por parte del terapeuta. Esta experiencia es algo habitual en la práctica clínica, como lo es también la de no lograr "conectar". Lo que pretendo mostrar a continuación es que esta experiencia no puede deberse a una proyección de la propia vida síquica en el otro, o a una simulación o mímica refleja que permitiría "hacer como si sintiera lo que el otro siente".

\section{Análisis de las distintas maneras de entender la empatía}

Dan Zahavi expresa de este modo la falta de consenso respecto al concepto de empatía:

“No hay consenso con respecto a lo que es ni a su relación con el contagio emocional, mímica motora, compartir emocional, proyección imaginativa, cambio de perspectiva, distress empático, preocupación emocional ¿Es la empatía una cuestión de compartir los sentimientos de otro, cuidar de otros, ser afectado emocionalmente por los pensamientos experimentados de otros sin tener las mismas experiencias? ¿Es imaginarse a sí mismo en la situación del otro o imaginarse siendo otro en otra situación o simplemente hacer inferencias acerca de los estados mentales de otros? ¿La empatía implica sentir la misma emoción que detecto en otros? ¿Suprime la diferencia yo-otro?" (Zahavi, 2014b) 
Como un modo de sortear, por ahora, esta confusión intentaré hacer tres aclaraciones terminológicas básicas que permitan avanzar.

En primer lugar, el contagio emocional sería un capturar simple, en principio automático, del sentir de los demás y no tendría asociado ningún elemento imaginativo ni reflexivo. Esta captación se produciría de forma directa y quien padece el contagio emocional comparte la misma emoción del o de los otros sin necesariamente ser consciente de ello (Darwall, 1998; Battaly, 2014).

En segundo lugar, la empatía sería un conocer el estado mental de otro sin que por ello se compartan necesariamente sentimientos (Darwall, 1998; Smith, 2014). Esta forma de conceptualizar la empatía no excluye, en principio, procesos imaginativos ni de espejamiento o mirroring ni tampoco el de adoptar la perspectiva del otro. Es decir, no dice nada respecto a la forma por la que se llega a ese conocimiento.

En tercer lugar, la simpatía sería una forma de "sentir por o junto con otros", es decir el simpatizante siente algún afecto o emoción semejante a la del estado de conciencia original o sujeto target; en contraste con la empatía, que cuando involucra conocer sentimientos, sería un "sentir con otros", como acompañando al target desde fuera (Smith, 2014). Es decir, la noción de simpatía supondría un componente emotivo o de "calidez" que no posee necesariamente el término empatía (Darwall, 1998; Coplan \& Goldie, 2014). Las definiciones de David Hume y Adam Smith apuntan también en la misma dirección, es decir, hacia un "sentir lo que otro siente" (Prinz, 2011).

Dentro de estas aclaraciones terminológicas conviene apuntar que, tal como explica Zahavi, existe un debate acerca del modo en el que se relacionan estas tres nociones. Por ejemplo, Darwall, piensa que el contagio emocional es una forma mínima de empatía (Darwall, 1998), mientras que Goldman niega que formas mínimas de empatía como las que fundamentan las neuronas espejo puedan estar en línea continua con fenómenos de empatía de "alto nivel"o empatía reconstructiva (Goldman, 2014). Algunos autores piensan que a la empatía le corresponde una preocupación por el bienestar del otro o preocupación empática, (Batson et al., 1991), mientras que para Prinz, la empatía no motiva preocupación alguna (Prinz, 2011). Así, Darwall, desarrolla más bien la preocupación simpática (sympathetic concern), que implica una preocupación por el bien de otro que podríamos llamar "emotiva" (Darwall, 1998).

\section{Tres teorías acerca de la empatía}

Asimismo, existen tres teorías que intentan explicar cómo se produce el conocimiento del estado mental de otro: la "teoría-teoría", la teoría por simulación y la propuesta fenomenológica. Las teorías por analogía o, actualmente "teoría-teoría" afirman que el conocimiento de los estados mentales de otros se produce por medio de un predecir racional, una inferencia acerca de ellos.

En oposición a lo anterior surgieron las teorías por simulación que postulan que el conocimiento de otros se produce al imaginarse el estado mental de otro y simularlo en mí.

Lo que está de fondo en ambas teorías es la idea de que no es posible acceder directamente a los estados mentales de otros, es decir, que lo máximo a lo que podemos aspirar en la comprensión de otros es a simular o imaginar sus estados mentales sin jamás salir verdaderamente del círculo de los propios.

En contraste con esta suposición, se encuentra la propuesta de la fenomenología, representada principalmente por Edmund Husserl (1859-1938) y Edith Stein (1891-1942). Estos filósofos estaban ocupados con el "problema de otras mentes", es decir con la forma en que conocemos las vivencias de otros sujetos. La respuesta estándar en el tiempo de Husserl eran las teorías del razonamiento por analogía a lo que el filósofo responde con la solución de la empatía. La empatía sería un modo de conciencia por el cual accedemos directamente a la experiencia de otros, a sus pensamientos, emociones y deseos. Nos capacita para experimentar al otro como alguien cuyos estados mentales tienen un objetivo, como minded. La empatía sería una forma de percepción, como puede ser la percepción de una mesa, en la que, sin embargo, el objeto no se nos da originariamente y además se nos da como perteneciente a otro.

No se trata de captar solo los estados emocionales de otros, sino de tener un acceso a la "vida mental" de otro, a su forma de manifestarse en la conducta y en las acciones. La empatía sería la experiencia de la "mente corporeizada" o encarnada.

Como se mencionó, tanto la teoría-teoría como la teoría por simulación niegan un acceso real a las vivencias del otro. En efecto, en la teoría-teoría lo que hay es una inferencia, que en realidad, tiene su inicio y fin en la propia vida mental. Del mismo modo, en la teoría por simulación, la inferencia es reemplazada por un proceso menos cognitivo, pero que termina en producir en sí mismo unos sentimientos o vivencias que no tienen por qué tener alguna relación con las vivencias del otro. Con esto, la posibilidad de proyectar la propia vida síquica en el otro es evidentemente muy alta. En cambio, la propuesta fenomenológica apuesta por un acceso directo a las vivencias del otro. En mi opinión solo un conocimiento de este tipo puede dar cuenta del proceso de comunicación que 
se da entre un buen terapeuta y su paciente. Ahondaré, pues, en la propuesta fenomenológica siguiendo al filósofo danés Dan Zahavi.

\section{La propuesta fenomenológica}

La fenomenología concibe los fenómenos de nuestra conciencia como referidos siempre a algo diferente de ella, y esto es lo que se quiere decir con la expresión "intencionales", pero hay que diferenciar las distintas formas en que algo se da ante la conciencia, del objeto real. Zahavi dice:"tenemos que diferenciar entre pensar en un león, imaginar un león y ver un león". En los tres casos aquello a lo que se dirige la conciencia es el león, pero el acto con el cual se dirige a ese objeto es distinto. No es lo mismo pensar en el sufrimiento de otro, imaginar el sufrimiento de otro y encontrarse directamente con el sufrimiento del otro en un contacto "cara a cara". Es a esta tercera forma de "contacto" de la conciencia con el objeto a lo que Zahavi llama empatía. Con esto no está negando que pensar en o imaginar el sufrimiento de otro no sean formas de conocimiento de ese sufrimiento, solo que son vías diferentes, fenómenos diferentes, incluso, problemas filosóficos y biológicos diferentes. Hay investigadores que han enfatizado el compartir emocional como condición necesaria de la empatía, compartir que implica no únicamente que ambas personas sientan algún tipo de emoción, sino que esas emociones sean congruentes o"la misma emoción". La diferencia con lo que afirma Zahavi es que la empatía se trata en primer lugar de percibir, sin que el sentir la emoción, sea condición necesaria de ese percibir. Puedo percibir la alegría por el éxito de alguien y sentir envidia por ello, pero mi envidia no disminuye en nada mi percepción de la alegría del otro. A la vez, puede ser que la alegría de otro no suscite emoción alguna en mí. La propuesta de Zahavi es la siguiente:

“Empatía es la experiencia de la mente corporeizada de otro, una experiencia que, más que eliminar la diferencia entre la propia experiencia y la experiencia ajena, considera la asimetría como un hecho (fact) necesario y persistente" (Zahavi 2014b)

La mente corporeizada es la traducción desde el inglés de "embodied mind". Corporeizado se refiere al hecho de que nuestro cuerpo actúa en el mundo, que no es una cosa como lo puede ser una piedra. En la idea de la mente corporeizada se rechaza decididamente el concepto cartesiano de "cuerpo humano", despojado de alma, reducido a una máquina que más que tener finalidad, tiene eficiencia (Lázaro 2014), que además está completamente separado del alma (Descartes 2002). Citando a Husserl, Zahavi dice que el cuerpo del otro se da como una unidad expresiva desde un inicio (Zahavi 2104b) y que sus gestos y expresiones faciales están imbuidas de significado para mí. Es decir, lo que ocurre en nosotros cuando vemos a alguien sonreír y captamos que está expresando alegría no es un proceso en pasos sucesivos por el cual A) primero veo cambios en la expresión facial, para después en un segundo paso B) inferir por medio de la percepción de esas contracciones que esos gestos significan "sonreír", para por último C) concluir que el otro está alegre. No, captamos, de algún modo, directamente la alegría. Asimismo, ¿Cuál es la diferencia que se da entre la propia experiencia y la experiencia ajena? Aunque el acto de empatizar se da como un dato originario al observador del estado mental del otro, la experiencia empatizada sigue siendo, en todo momento, una experiencia cuya originalidad solo puede pertenecer al sujeto target. Esa experiencia jamás se da a la conciencia del observador de la misma manera en que se le da al que experimenta "de primera mano" el estado mental. El sufrimiento por la pérdida de un ser querido de otra persona no se da exactamente como si yo fuera el que está viviendo esa experiencia, por muy cercano que me sea quien lo está viviendo. Incluso, si quien murió es mi padre, el dolor de mi hermano y el mío, nunca serían el mismo dolor.

Esta diferencia es necesaria porque si no fuera así, la experiencia psicológica sería que él y yo somos la misma persona, algo que, en sí, consideramos incluso patológico. Pero además es necesaria porque justamente en ello radica lo sui generis de este tipo de percepción: en que percibo el estado mental de otro como del otro.

\section{Discusión}

La propuesta fenomenológica no niega que la imaginación, la contextualización y la inferencia puedan jugar un rol importante en la captación de la vida síquica de otros (sobre todo en lo que se refiere a la profundidad de tal captación). A mi juicio, su ventaja es que trata los estados mentales de otros como el dato primario al que cualquier inferencia o proceso imaginativo debe, en último término, atenerse. Por ejemplo, en el contexto de la relación terapeuta-paciente, si percibo empáticamente que el paciente está triste por algo, mi interpretación de su estado mental no está autorizada a llegar a la conclusión de que está alegre. Asimismo, tampoco podría prescindir completamente de la experiencia empática si quiero comprender de verdad, cómo acepta el paciente su reciente diagnóstico de cáncer en etapa avanzada. Sin embargo, tampoco puedo suponer que la empatía me dice todo respecto a la experiencia de quien tengo enfrente de mí. Para profundizar en ella se requieren otros procesos perceptivos y cognitivos. Pero, ¿por qué darle tanta importancia a comprender el estado mental de los pacientes? En la medicina moderna tenemos muchas herramientas para el diagnóstico y tratamiento que no requieren de este conocimiento para ser efectivas, por ejemplo, la telemedicina, la medicina basada en evidencia, las guías clínicas, procesos altamente 
tecnificados, etc. ¿Por qué aumentar la carga y la responsabilidad en los profesionales de salud, ya suficientemente sobrecargados, con "habilidades blandas" menos efectivas que un examen de laboratorio para diagnosticar y tratar una enfermedad? Y aún si se le diera importancia a la empatía, el asumir que esta sea una forma sui generis de percibir, tal como se afirma en este trabajo, es problemático. El simular el estado mental de otro como una mímica o el hacer una teoría acerca de lo que el otro siente, parecen destrezas que podrían enseñarse en un taller, pero, ¿es posible enseñar a percibir directamente el estado mental de otro? En este trabajo he afirmado que de hecho lo hacemos, incluso involuntariamente, pero esa percepción podría no tener ninguna influencia en el trato con el paciente o producir sentimientos negativos en el terapeuta (por ejemplo, si percibo rabia en el paciente y eso despierta resentimiento). Así, la utilidad y la necesidad de desarrollar esta facultad solo puede entenderse en un marco más amplio, que tiene que ver con aquello que entendemos cómo cuidar profesionalmente de personas que poseen libertad, valores, historia y dignidad y no solo una dimensión biológica para la cual el objetivo de curar podría ser suficiente. Un cuidar profesional que se comprende como todo aquello que se realiza por el genuino interés por el bien integral del paciente y no solo por la preocupación por sus funciones fisiológicas, no puede dejar de lado facultades como la empatía.

\section{Conclusión}

La empatía es una facultad que permitiría el acceso directo -pero no por eso completo o absoluto- a las vivencias mentales de otros. Dicha forma de comprender la noción tiene puntos de contacto con las descripciones que autores como Carl Rogers o Nel Noddings han hecho del término $y$, sobre todo, son congruentes con formas de comprender el cuidado que engloban más que el aspecto biológico. Por lo tanto, una primera conclusión es que la empatía, tal como se propone desde la fenomenología es un elemento relevante en el cuidado profesional, entendido como todo aquello que se realiza por el genuino interés por la persona toda. Si esto es así, sería deseable el saber cómo desarrollarla en la educación médica. Para esto se requiere reflexionar acerca de qué significa que podamos conocer estados mentales ajenos de modo directo, qué en nuestra interioridad es la condición de posibilidad para dicha facultad. Este importante problema excede a los límites del presente trabajo, por lo que solo mencionaré que parece poco probable que una educación médica centrada solo en el análisis de datos biológicos pueda dar cuenta del desarrollo de este tipo de habilidades.

Una segunda conclusión es que, a pesar de que los paradigmas de cuidado mencionados, sitúan a la empatía como lo central en el cuidado profesional, dado el análisis realizado, parece cuestionable es afirmar que dicha facultad pueda erigirse como la actitud básica que deba poseer el terapeuta para lograr un trato digno hacia su paciente. En primer lugar, debido a que cualquier facultad puede ser mal utilizada y en particular la empatía, podría servir para dominar y manipular al otro. En segundo lugar, porque la facultad es solo una herramienta que permite tomar decisiones personales frente a situaciones particulares. El desarrollo de la empatía en un terapeuta no "produce" de por sí, buenas decisiones y actitudes. Estas provienen de una instancia antropológica más profunda y más personal que la de la esfera de las facultades. Esta instancia antropológica es aquella en la cual reside nuestra tendencia al bien y, sobre todo, nuestra tendencia al bien de otras personas. El cuidado profesional, en última instancia, solo puede y debe fundarse en una tendencia voluntaria de búsqueda del bien de otro: el paciente que tengo frente a mí, aquí y ahora.

\section{Fuentes de financiamiento}

Sin fuentes de financiamiento.

\section{Contribuciones y conflictos declarados por los autores}

Sin conflictos de interés

\section{Referencias}

Batson, C. D., Batson, J. G., Slingsby, J. K., Harrell, K. L., Peekna, H. M., \& Todd, R. M. (1991). Empathic joy and the empathy-altruism hypothesis. Journal of Personality and Social Psychology 61, 413-426.

Battaly, H. (2014). Is Empathy a Virtue? En A. Coplan \& P. Goldie (Eds.), Empathy:Philosophical and psychological perspectives. Oxford University Press, Oxford.

Bloom, P. (2018). Against Empathy. Random House Uk, UK.

Coplan, A., \& Goldie, P. (2014). Empathy: Philosophical and Psychological Perspectives (Edición: Reprint). OUP Oxford, Oxford.

Darwall, S. (1998). Empathy, Sympathy, Care. Philosophical Studies: An International Journal for Philosophy in the Analytic Tradition 89, 261-282.

Descartes, R. (2002). El Tratado del hombre: Tratado de las pasiones (G. Quintás, Trad.). RBA Coleccionables, Barcelona.

di Pellegrino, G., Fadiga, L., Fogassi, L., Gallese, V., \& Rizzolatti, G. (1992). Understanding motor events: A neurophysiological study. Experimental Brain Research 91, 176-180. 
Fricchione, G. (2017). Separation-Attachment Theory in Illness and the Role of the Healthcare Practitioner. En T. A. Hutchinson (Ed.), Whole Person Care: Transforming Healthcare. Springer International Publishing, Cham.

Hutchinson, T. A. (Ed.). (2017). Whole Person Care: Transforming Healthcare. Springer International Publishing, Cham.

Lázaro Cantero, R. (2012). Descartes y las pasiones del alma: La Filosofía de las pasiones y la Escuela de Salamanca. Descartes and the Passions of the Soul: The philosophy of passions and the School of Salamanca, 249.

Mitereva, G. A. (1945). Caring for mother and child-The most important national objective. Feldscher Akush, 1, 1-9.

Noddings, N. (2013). Caring: A Relational Approach to Ethics and Moral Education (Second Edition, Updated edition). University of California Press, Berkeley, Calif.
Prinz, J. (2011). Against Empathy. The Southern Journal of Philosophy 49, 214-233.

Puchalski, CM, Vitillo, R. Hull SK \& Reller, N. (2014). Improving the spiritual dimension of whole person care: Reaching national and international consensus. Journal of Palliative Medicine 17, 642-656.

Rogers, CR. (1957). The necessary and sufficient conditions of therapeutic personality change. Journal of Consulting Psychology 21, 95-103.

Smith, M. (2014). Empathy, Expansionism, and the Extended Mind. En A. Coplan \& P. Goldie (Eds.), Empathy: Philosophical and psychological perspectives. Oxford University Press, Oxford.

Zahavi, D. (2014a). Empathy and Other-Directed Intentionality. Topoi 33, 129-142.

Zahavi, D. (2014b). Self and other: Exploring subjectivity, empathy, and shame. Oxford University Press, Oxford. 